\title{
First Diagonal Branch Artery
}

National Cancer Institute

\section{Source}

National Cancer Institute. First Diagonal Branch Artery. NCI Thesaurus. Code C102297.

The first artery arising from the left anterior descending (LAD) artery that supplies the anterolateral wall, when counted from proximal to distal. 